\title{
Endoscopic Ultrasound-Guided Fine-Needle Biopsy As A Valuable Tool for Studying The Intra-Tumoral Microbiome in Pancreatic Ductal Adenocarcinoma
}

\section{Chia-Sheng Chu}

China Medical University Hospital

\section{Chi-Ying Yang}

China Medical University Hospital

Chun-Chieh Yeh

China Medical University Hospital

Ro-Ting Lin

China Medical University

Chi-Ching Chen

China Medical University Hospital

Li-Yuan Bai

China Medical University Hospital

Mien-Chie Hung

China Medical University

Chun-Che Lin

China Medical University Hospital

Chun-Ying Wu

National Yang Ming University Hospital

Jaw-Town Lin ( $\square$ jawtown@gmail.com )

China Medical University Hospital

\section{Research Article}

Keywords: pancreatic ductal adenocarcinoma, intra-tumoral microbiome, microbiome, endoscopic ultrasound-guided fine needle biopsy, next generation sequencing

Posted Date: September 9th, 2021

DOI: https://doi.org/10.21203/rs.3.rs-876384/v1

License: (a) (i) This work is licensed under a Creative Commons Attribution 4.0 International License.

Read Full License 


\section{Abstract}

\section{Introduction:}

A new approach by investigating the intra-tumoral microbiome raised great interest because they may influence the host immune response and natural history of the disease. However, previous studies on the intra-tumoral microbiome of pancreatic ductal adenocarcinoma (PDAC) were mostly based on examining the formalin-fixed paraffin-embedded tumor specimens. This study aims to investigate the feasibility of using endoscopic ultrasound-guided fine-needle biopsy (EUS-FNB) as a complementary procedure of surgical biopsy to obtain adequate fresh pancreatic cancer tissue for intra-tumoral microbial research.

\section{Materials and methods:}

This was a prospective pilot study performed at a single tertiary referral center. We obtained pancreatic cancer tissue by EUS-FNB and surgical biopsy, respectively. We amplified the V3-V4 hyper-variable region of bacterial 16S ribosomal ribonucleic acid (rRNA) genes, constructed a pair-end library, and performed high-throughput sequencing.

\section{Results:}

From August 2020 to November 2020, nine eligible patients with PDAC were enrolled in this study. The intra-tumoral microbiome profile was successfully generated from the PDAC cancer tissue obtained by EUS-FNB as well as by surgical biopsy. There was no significant difference in intra-tumoral alphadiversity or bacterial taxonomic composition between tissues obtained by EUS-FNB and by surgical biopsy.

\section{Conclusion:}

EUS-FNB can collect sufficient fresh cancer tissue for microbiome analyses without complication. The intra-tumoral microbiome profile in tissues obtained by EUS-FNB had similar alpha-diversity and taxonomic profiles with those obtained by surgical biopsy. It implicated, except for surgical biopsy, EUSFNB can be another valid and valuable tool for studying intra-tumoral microbiome in patients with resectable and unresectable PDAC.

\section{Introduction}

In recent years, there was a remarkable increase in the number of studies investigating the gut microbiome and cancer. It ranged from oncogenesis, cancer progression, outcome prediction to resistance to anticancer therapies. ${ }^{1-3}$ Moreover, cancer patients seem to harbor a specific microbiome 
composition in the tumor niche which differs from healthy controls. ${ }^{4-7}$ Nejman et al. found that intratumoral microbiome composition is diverse and cancer type-specific. ${ }^{4}$ Riquelme et al. disclosed the intratumoral microbiome composition of pancreatic ductal adenocarcinoma (PDAC) patients. They identified a specific intra-tumoral microbiome signature predicting the long-term survivorship of PDAC. ${ }^{6}$ However, studies on the intra-tumoral microbiome of PDAC were mostly based on examining the formalin-fixed paraffin-embedded (FFPE) tumor specimens obtained from patients who underwent surgical resection. 4,6 The risk of contamination with the environmental microbiota can hardly be avoided when handling the FFPE tumor specimens in a retrospective way. Ensuring that fresh tumor tissues are obtained in a sterile way is the basis of microbial research of PDAC.

PDAC is usually diagnosed late or detected until with metastases, and only a small proportion of PDAC patients can receive curative surgery. Therefore, most PDAC patients cannot provide fresh and sufficient cancer tissue for microbial studies. Recently, endoscopic ultrasound-guided fine-needle biopsy (EUS-FNB) has assumed a growing role in the diagnosis and management of PDAC. ${ }^{8,9}$ It can usually provide sufficient materials for cytological and histological examination of cancerous tissue in patients with unresectable PDAC. ${ }^{10,11}$

Whether EUS-FNB can be used as a complementary procedure to obtain adequate fresh pancreatic cancer tissue to investigate the intra-tumoral microbiome remained unclear. Therefore, we conducted a prospective study to investigate the intra-tumoral microbiome profile of pancreatic cancerous tissue obtained by EUS-FNB and surgical biopsy. We compared the yield rates, adverse events, and complications between these two procedures.

\section{Materials And Methods}

This was a prospective pilot study performed at a single tertiary referral center (China Medical University Hospital, Taichung, Taiwan). All EUS-FNB procedures and surgical operations were performed at China Medical University Hospital in accordance with the guideline of European Society of Gastrointestinal Endoscopy and operative regulations, respectively. The patient considered eligible for this study included patients with suspected pancreatic cancer by computed tomography or magnetic resonance imaging. Patients were excluded if they were unable to provide informed consent or were using antibiotics or probiotics before the procedure. Written informed consent was obtained from each patient or family. This study was approved by the Institutional Review Boards, China Medical University Hospital. (CMUH109REC3-026).

\section{Human Tumor Specimens:}

During the surgical operation, we performed an ultrasound-guided core-needle biopsy for the pancreatic cancerous tissue with a 14-gauge needle. All surgical specimens were sterilely immersed in the lysis buffer containing $2.5 \%$ tris- $\mathrm{HCl}, 2.0 \%$ EDTA, $0.5 \%$ sodium dodecyl sulfate, and $95 \%$ distilled $\mathrm{H}_{2} \mathrm{O}$, and were immediately sent for microbial analysis. 
EUS-FNB was performed by an experienced endoscopist using a linear array echoendoscope (Olympus GF-UCT260, Olympus Medical Systems, Tokyo, Japan) while the patient was conscious sedated. The pancreatic lesion was carefully examined to assure no major vessels within the needle pathway before puncture in color Doppler mode. A 22-gauge needle (Acquire, Boston Scientific Corporation, Natick, Massachusetts, United States) was used for tissue sampling. The pancreatic lesion was identified and then punctured under EUS guidance. When the needle was inserted into the lesion, the stylet was slowly withdrawn. The FNB specimens were collected without negative pressure after 20 to 40 back-and-forth movements by fanning technique. In the first pass, the tissue specimens were immediately immersed in the lysis buffer, and it was delivered immediately for microbial analysis. In the second and third passes, the tissue specimens were collected and fixed with $10 \%$ neutral buffered formalin for histological examination.

\section{DNA extraction, bacterial 16S rRNA sequencing and microbiome analysis}

Tumor samples were kept on ice and transferred to a laboratory for DNA extraction in accordance with manufacturer's protocol, which was done by using Real Genomics DNA Extraction Kit YGE100R (RBC Bioscience Corp., New Taipei City, Taiwan). The isolated DNA aliquot was stored at $-80^{\circ} \mathrm{C}$ before $16 \mathrm{~s}$ ribosomal ribonucleic acid (rRNA) gene sequencing. DNA concentration and quality were evaluated by NanoDrop ND-1000 (Thermo Scientific, Wilmington, DE, USA). The hypervariable region V3-V4 of bacterial $16 \mathrm{~S}$ rRNA genes was amplified by polymerase chain reaction using bar-coded universal primers $341 \mathrm{~F}(\mathrm{~F}$, forward primer; 5-CCTACgggNggCWgCAg-3') and 805R (R, reverse primer; 5'-gACTACHCgggTATCTAATCC$\left.3^{\prime}\right)$. Library construction and sequencing of amplicon DNA samples were committed to Germark Biotechnology (Taichung, Taiwan). A pair-end (2x300) library (insert size of 465 base pairs for each sample) was constructed with TruSeq Nano DNA Library Prep kit (Illumina, San Diego, CA, USA), and high-throughput sequencing was performed on an Illumina MiSeq 2000 sequencer with MiSeq Reagent Kit v3 (Illumina). The bioinformatics analysis of $16 \mathrm{~S}$ rRNA amplicon was conducted by Germark Biotechnology (Taichung, Taiwan). Briefly, on a per-sample basis, paired-end reads were merged using USEARCH (v8.0.1623), ${ }^{12}$ with a minimum overlap of read pair set at 8 base pairs (bp). Merged reads were quality-filtered with Mothur (v1.34.1) ${ }^{13}$ to remove reads shorter than $400 \mathrm{bp}$ or longer than $550 \mathrm{bp}$, as well as reads with a minimum average quality score lower than 27 . In addition, reads containing an ambiguous base or homopolymer exceeding $8 \mathrm{bp}$ were excluded. Chimera detection was performed using USEARCH (reference mode and 3\% minimum divergence) and removed from further analysis. Qualityfiltered and non-chimeric reads were analyzed (UPARSE pipeline) ${ }^{14}$ to generate operational taxonomic units (OTUs) per sample (at 97\% identity level). The OTU representative sequences were searched against the Greengenes 13_5 database by using USEARCH global alignment to identify the corresponding taxonomy of the best hit. OTUs without a hit or with only a weak hit, that is, the function "(\% sequence identity + \% alignment coverage) $/ 2$ " less than $93,{ }^{15}$ was excluded from the following analysis. Diversity indices (e.g., Shannon, Simpson, Inv Simpson) were estimated with the R package phylosea. ${ }^{16}$

\section{Statistical analyses:}


The alpha-diversity in the tissues obtained by EUS-FNB and surgical biopsy was compared using the Mann-Whitney test. Hierarchical clustering (via complete-linkage algorithm) of microbiomes was conducted using the Bray-Curtis distance of OTU-level relative abundance profile, based on which principal coordinates analysis (PCoA) was also performed using the R package ade $4 .{ }^{17} \mathrm{~A} p$-value less than 0.05 was considered statistically significant.

\section{Results}

From August 2020 to November 2020, nine eligible patients with PDAC were enrolled in this study. They were six men and three women, with a mean age of 61.8 (47-76) years. Five patients had PDAC located in the head of pancreas, one in the body, and three in the tail. Five PDAC patients were diagnosed with stage III and four with stage IV. We used EUS-FNB to obtain pancreatic cancer tissues from six patients. We obtained tissue samples from four patients during surgery. One patient underwent both EUS-FNB and surgery. This patient underwent EUS-FNB, and the result revealed atypical glands initially; the patient was later confirmed as adenocarcinoma by a subsequent surgical operation. Five patients $(5 / 6,83.3 \%)$ who underwent EUS-FNB were confirmed PDAC, while all four patients $(4 / 4,100 \%)$ in the surgical group were diagnosed as PDAC. There was no internal bleeding, pancreatitis, and other adverse events after EUSFNB. No internal bleeding nor other complications occurred in subjects who underwent surgery.

The intra-tumoral microbiome profile was successfully generated from the PDAC cancer tissue obtained by EUS-FNB as well as by surgical biopsy. For microbial profiling, a total of 1.2 million pair-end reads were generated, of which 962 thousand reads passed quality filtering and were not chimera. To determine microbial diversity and composition, reads were aligned to the Greengenes database, and non-bacterial sequences were removed. The number of observed OTUs and the intra-tumoral bacterial diversity (alphadiversity, represented by Shannon, Simpson, and inverse Simpson indices) were not significantly different between the two groups (Fig. 1), indicating the intra-tumoral bacterial abundances were similar between EUS-FNB and surgical groups. PCoA also revealed no significant difference in bacterial OTU composition between EUS-FNB and surgical biopsy $(P=0.085)$ (Fig. 2). In other words, the intra-tumoral bacterial composition between these two groups were close.

\section{Discussions}

The associations of gut microbiota with various human diseases and various types of cancers have been widely investigated, especially their roles in tumorigenesis and cancer progression. The microbiome has emerged as a new potential biomarker for cancer diagnosis, risk stratification, and prognosis. Previous studies usually investigated the association between the gut microbiome and PDAC by collecting the fecal ${ }^{3,18-20}$ or salivary ${ }^{21-23}$ samples to determine the microbial profile. Some bacteria, such as Porphyromonas, Fusobacterium, Aggregatibacter, Prevotella, and Capnocytophaga, were found to play a role in the development of PDAC. ${ }^{24}$ However, the microbiome profiles obtained from saliva and feces 
were inconsistent and conflicting, probably due to various technique of sample extraction, processing, and data analysis. ${ }^{25}$

Recently, a new approach by investigating the intra-tumoral microbiome raise great interest. Geller et al. showed the presence of Gammaproteobacteria in PDAC might be responsible for the tumor resistance to gemcitabine. ${ }^{5}$ The gut microbiome also plays a significant role in antitumor immune responses and predicts the efficacy of immune-checkpoint inhibitors in cancer patients. ${ }^{26,27}$ Riquelme et al. identified an intra-tumoral microbiome signature (Pseudoxanthomonas-Streptomyces-Saccharopolyspora-Bacillus clausii) which was predictive of long-term survivor in PDAC. They demonstrated that PDAC microbiome composition, which cross-talked to the gut microbiome, could influence the host immune response and natural history of the disease. ${ }^{6}$ Chakladar et al. outlined the intra-tumoral microbiome of 187 PDAC samples through large-scale sequencing data from The Cancer Genome Atlas (TCGA), and they found potentially cancer-promoting or immune-inhibiting microbes - most of them belonged to Proteobacteria phylum. ${ }^{28}$ Nejman et al. reported that intra-tumoral microbiome composition is diverse and cancer typespecific. They analyzed the intra-tumoral microbiome of 1,526 samples from seven human tumor types, including breast, lung, ovary, pancreas, melanoma, bone, and brain tumors. Bacteria belonging to the Firmicutes and Bacteroidetes phyla were the most abundant species in colorectal tumors, while Proteobacteria dominated the microbiome of PDAC. ${ }^{4}$

However, previous studies on the intra-tumoral microbiome of PDAC were mostly based on the FFPE tumor specimens obtained during surgical resection. ${ }^{4,6}$ The majority of PDAC patients were diagnosed at advanced stages and thus precluded surgical resection. Masi et al. compared the results of microbiome profile in FFPE specimens obtained by surgical biopsy and EUS-FNB using Decontam ${ }^{29}$ (http://github.com/benjjneb/decontam), an open-source R package, to remove contaminant DNA sequences. They found there was no significant difference in alpha-diversity, beta-diversity, or taxonomic profiles between EUS-FNB and surgical biopsy in three patients with matched samples. ${ }^{30}$ They claimed that EUS-FNB could substitute surgical biopsy in the PDAC tissue sampling for microbial research. This prospective study confirmed that both two methods can collect sufficient cancer tissue for microbiome analyses.

In conclusion, EUS-FNB can collect sufficient fresh cancer tissue for microbiome analyses without complication. The intra-tumoral microbiome profile from EUS-FNB had similar alpha-diversity and taxonomic profiles with surgical biopsy. It implicated, except for surgical biopsy, EUS-FNB can be another valid and valuable tool for studying intra-tumoral microbiome in patients with resectable and unresectable PDAC.

\section{Abbreviations}

pancreatic ductal adenocarcinoma $=$ PDAC, formalin-fixed paraffin-embedded $=F F P E$, endoscopic ultrasound-guided fine-needle biopsy = EUS-FNB, ribosomal ribonucleic acid = rRNA, base pairs = bp, 
operational taxonomic unit $=$ OTU, principal coordinates analysis $=P C o A$

\section{Declarations}

\section{Acknowledgements}

This work was supported in part by China Medical University (DMR 109-215) and the Ministry of Science and Technology, Taiwan (MOST 109-2327-B-039-001).

\section{Data sharing statement}

No additional data are available.

\section{AUTHOR CONTRIBUTIONS}

CS Chu was responsible for the study conception and design and preparing the initial draft of manuscript. RT Lin was responsible for data analysis and interpretation. CY Yang, CC Yeh, CC Chen, LY Bai, MC Hung and CC Lin participated in the study conception and data collections. CY Wu and JT Lin performed data analysis and interpretation as well as manuscript drafting and revision.

\section{Conflict-of-interest statement}

The authors declare no conflicts of interest related to this manuscript.

\section{References}

1. Meng, C., Bai, C., Brown, T. D., Hood, L. E. \& Tian, Q. Human gut microbiota and gastrointestinal cancer. Genomics Proteomics Bioinformatics, 16, 33-49 https://doi.org/10.1016/j.gpb.2017.06.002 (2018).

2. Goodman, B. \& Gardner, H. The microbiome and cancer. J Pathol, 244, 667-676 https://doi.org/10.1002/path.5047 (2018).

3. Pushalkar, S. et al. The pancreatic cancer microbiome promotes oncogenesis by Induction of innate and adaptive immune suppression. Cancer Discov, 8, 403-416 https://doi.org/10.1158/21598290.CD-17-1134 (2018).

4. Nejman, D. et al. The human tumor microbiome is composed of tumor type-specific intracellular bacteria., 368, 973-980 https://doi.org/10.1126/science.aay9189 (2020).

5. Geller, L. T. et al. Potential role of intratumor bacteria in mediating tumor resistance to the chemotherapeutic drug gemcitabine., 357, 1156-1160 https://doi.org/10.1126/science.aah5043 (2017).

6. Riquelme, E. et al. Tumor microbiome diversity and composition influence pancreatic cancer outcomes., 178, 795-806 e12https://doi.org/10.1016/j.cell.2019.07.008 (2019). 
7. Oliva, M. et al. Tumor-associated microbiome: where do we stand? Int J Mol Sci, 22, 1446 https://doi.org/10.3390/ijms22031446 (2021).

8. Crinò, S. F. et al. EUS-FNB with or without on-site evaluation for the diagnosis of solid pancreatic lesions (FROSENOR): protocol for a multicenter randomized non-inferiority trial. Dig Liver Dis, 51, 901-906 https://doi.org/10.1016/j.dld.2019.03.008 (2019).

9. Ishikawa, T. et al. Clinical impact of EUS-guided fine needle biopsy using a novel franseen needle for histological assessment of pancreatic diseases. Can J Gastroenterol Hepato/ 2019; 2019: 8581743. https://doi.org/10.1155/2019/8581743

10. Yang, Y. et al. Endoscopic ultrasound-guided fine needle core biopsy for the diagnosis of pancreatic malignant lesions: a systematic review and meta-analysis. Sci Rep, 6, 22978 https://doi.org/10.1038/srep22978 (2016).

11. Wani, S. et al. AGA white paper: optimizing endoscopic ultrasound-guided tissue acquisition and future directions. Clin Gastroenterol Hepatol, 16, 318-327 https://doi.org/10.1016/j.cgh.2017.10.020 (2018).

12. Edgar, R. C. Search and clustering orders of magnitude faster than BLAST., 26, 2460-2461 https://doi.org/10.1093/bioinformatics/btq461 (2010).

13. Schloss, P. D. et al. Introducing mothur: open-source, platform-independent, community-supported software for describing and comparing microbial communities. Appl Environ Microbiol, 75, 75377541 https://doi.org/10.1128/AEM.01541-09 (2009).

14. Edgar, R. C. UPARSE: highly accurate OTU sequences from microbial amplicon reads. Nat Methods, 10, 996-998 https://doi.org/10.1038/nmeth.2604 (2013).

15. Quast, C. et al. The SILVA ribosomal RNA gene database project: improved data processing and webbased tools. Nucleic Acids Res, 41 (Database issue), D590-D596 https://doi.org/10.1093/nar/gks1219 (2013).

16. McMurdie, P. J. \& Holmes, S. Phyloseq: an R package for reproducible interactive analysis and graphics of microbiome census data. PLoS One, 8, e61217 https://doi.org/10.1371/journal.pone.0061217 (2013).

17. Dray, S. \& Dufour, A-B. The ade4 package: implementing the duality diagram for ecologists. Journal of Statistical Software, 22, 1-20 https://doi.org/10.18637/jss.v002.i04 (2007).

18. Half, E. et al. Fecal microbiome signatures of pancreatic cancer patients. Sci Rep, 9, 16801 https://doi.org/10.1038/s41598-019-53041-4 (2019).

19. Ren, Z. et al. Gut microbial profile analysis by MiSeq sequencing of pancreatic carcinoma patients in China. Oncotarget, 8, 95176-95191 https://doi.org/10.18632/oncotarget.18820 (2017).

20. Qiu, L. et al. A comparative assessment of the influences of human impacts on soil Cd concentrations based on stepwise linear regression, classification and regression tree, and random forest models. PLoS One, 11, e0151131 https://doi.org/10.1371/journal.pone.0151131 (2016).

21. Fan, X. et al. Human oral microbiome and prospective risk for pancreatic cancer: a population-based nested case-control study., 67, 120-127 https://doi.org/10.1136/gutjnl-2016-312580 (2018). 
22. Michaud, D. S. et al. Plasma antibodies to oral bacteria and risk of pancreatic cancer in a large European prospective cohort study., 62, 1764-1770 https://doi.org/10.1136/gutjnl-2012-303006 (2013).

23. Farrell, J. J. et al. Variations of oral microbiota are associated with pancreatic diseases including pancreatic cancer., 61, 582-588 https://doi.org/10.1136/gutjnl-2011-300784 (2012).

24. Karpinski, T. M. The microbiota and pancreatic cancer. Gastroenterol Clin North Am, 48, 447-464 https://doi.org/10.1016/j.gtc.2019.04.008 (2019).

25. Sinha, R. et al. Assessment of variation in microbial community amplicon sequencing by the microbiome quality control (MBQC) project consortium. Nat Biotechnol, 35, 1077-1086 https://doi.org/10.1038/nbt.3981 (2017).

26. Routy, B. et al. Gut microbiome influences efficacy of PD-1-based immunotherapy against epithelial tumors., 359, 91-97 https://doi.org/10.1126/science.aan3706 (2018).

27. Gopalakrishnan, V. et al. Gut microbiome modulates response to anti-PD-1 immunotherapy in melanoma patients., 359, 97-103 https://doi.org/10.1126/science.aan4236 (2018).

28. Chakladar, J. et al. The pancreatic microbiome is associated with carcinogenesis and worse prognosis in males and smokers. Cancers (Basel), 12, 2672 https://doi.org/10.3390/cancers12092672 (2020).

29. Davis, N. M., Proctor, D. M., Holmes, S. P., Relman, D. A. \& Callahan, B. J. Simple statistical identification and removal of contaminant sequences in marker-gene and metagenomics data. Microbiome, 6, 226 https://doi.org/10.1186/s40168-018-0605-2 (2018).

30. Masi, A. C. et al. Endoscopic ultrasound (EUS)-guided fine needle biopsy (FNB) formalin fixed paraffin-embedded (FFPE) pancreatic tissue samples are a potential resource for microbiota analysis., https://doi.org/10.1136/gutjnl-2020-322457 (2020). gutjnl-2020-322457

\section{Figures}




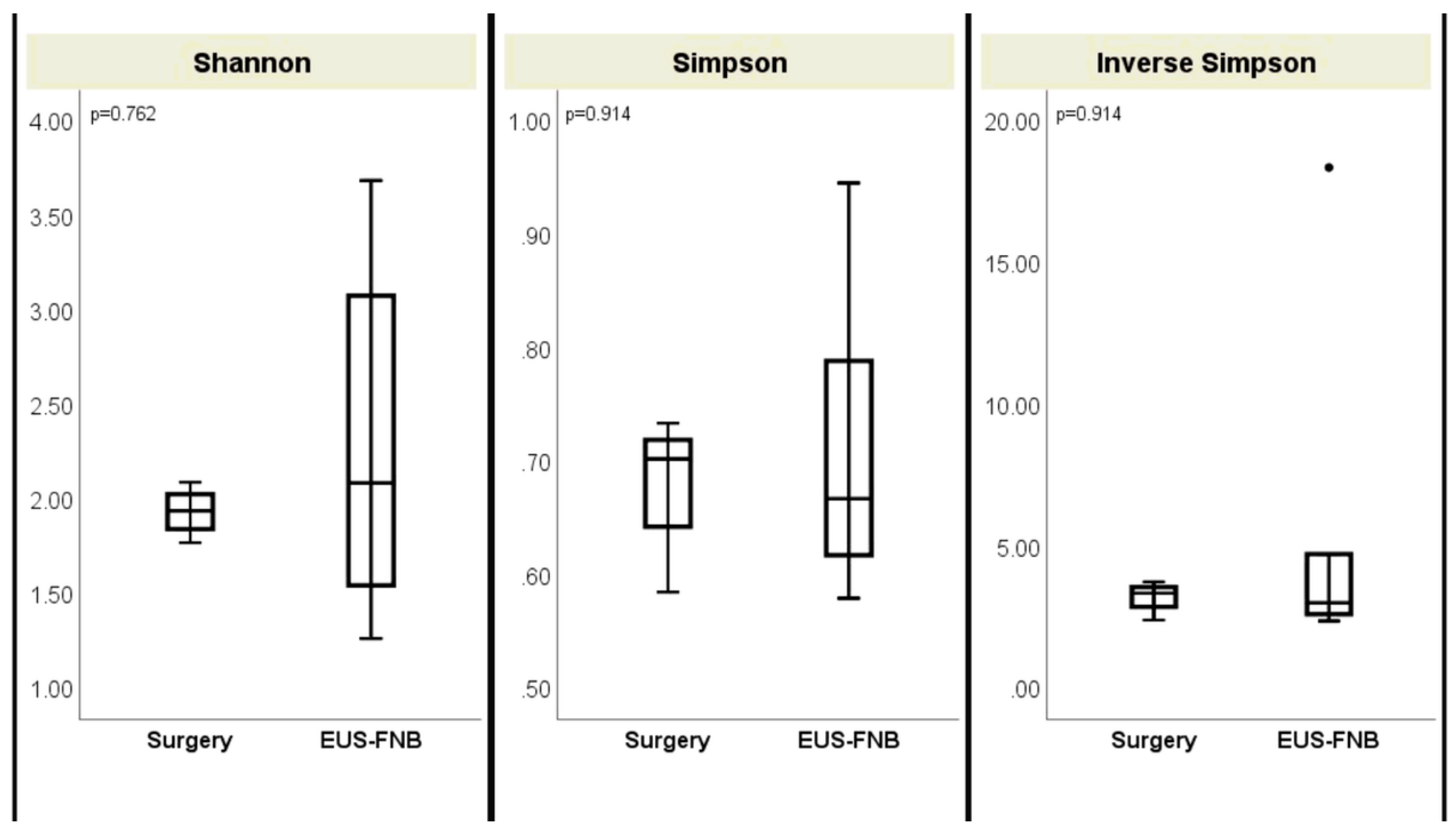

Figure 1

There was no significant difference in alpha-diversity (represented by Shannon, Simpson, and inverse Simpson indices) between endoscopic ultrasound-guided fine-needle biopsy (EUS-FNB) group and surgical group. 


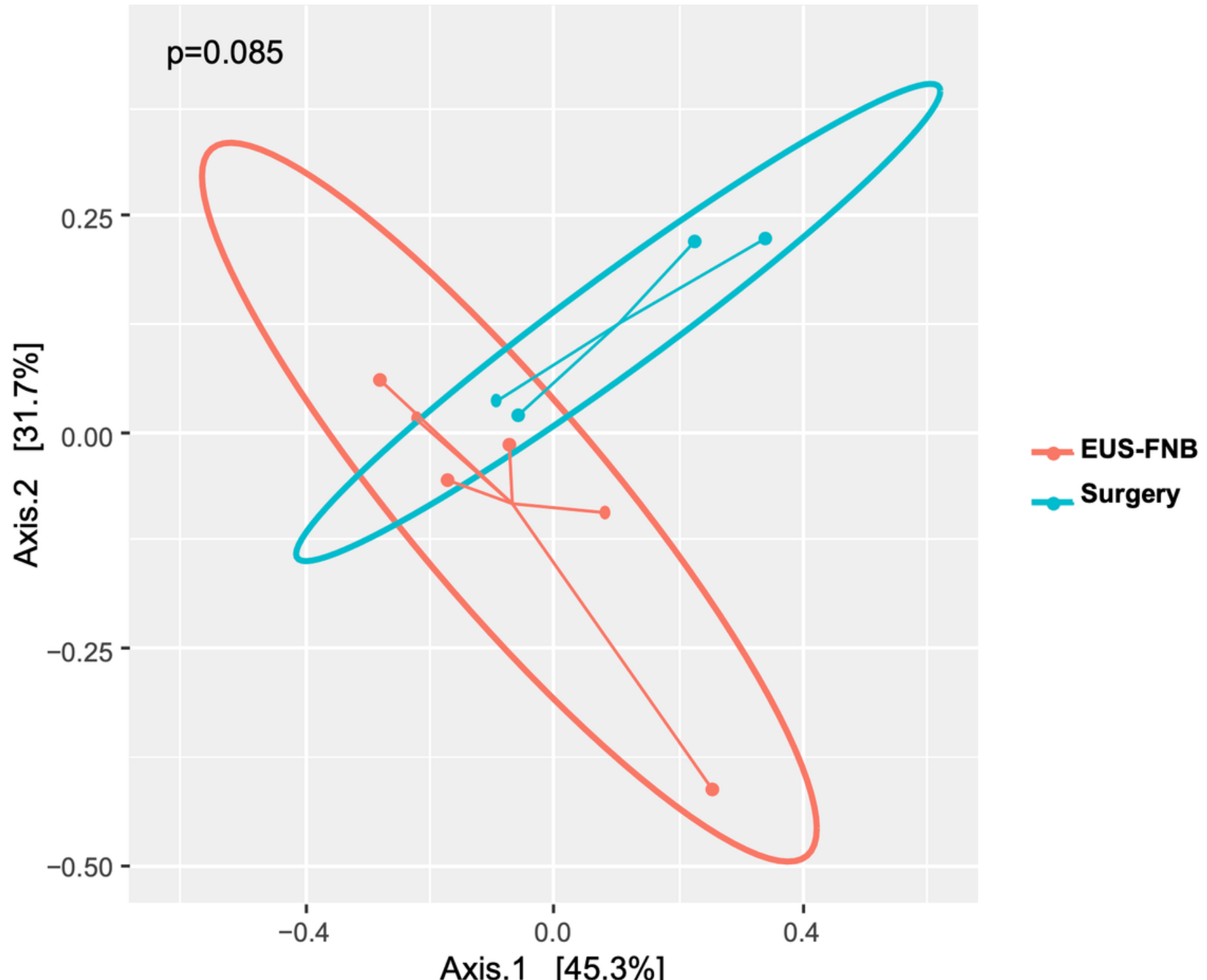

\section{Figure 2}

Principal coordinates analysis $(P C o A)$ revealed no significant difference $(P=0.085)$ in bacterial operational taxonomic unit (OTU) composition at genus level between endoscopic ultrasound-guided fine-needle biopsy (EUS-FNB) group and surgical group. 